\title{
Commissioning of the BRIKEN beta-delayed neutron detector for the study of exotic neutron-rich nuclei
}

\begin{abstract}
A. Tolosa-Delgado ${ }^{1, \star}$, J. Agramunt ${ }^{1}$, D. S. Ahn ${ }^{2}$, A. Algora ${ }^{1,12}$, H. Baba ${ }^{2}$, S. Bae ${ }^{3}$, N. T. Brewer ${ }^{4}$, R. Caballero Folch ${ }^{5}$, F. Calvino ${ }^{6}$, P. J. Coleman-Smith ${ }^{7}$, G. Cortes $^{6}$, T. Davinson ${ }^{9}$, I. Dillmann ${ }^{5}$, C. Domingo-Pardo ${ }^{1}$, A. Estrade ${ }^{10}$, N. Fukuda ${ }^{2}$, S. Go ${ }^{11,2}$, C. J. Griffin ${ }^{9}$, R. Grzywacz ${ }^{11}, J \mathrm{Ha}^{3}, \mathrm{O}$. Hall $^{9}$, L. Harkness-Brennan ${ }^{8}$, T. Isobe ${ }^{2}$, D. Kahl ${ }^{2}$, G. G. Kiss ${ }^{2,12}$, M. Kogimtzis ${ }^{7}$, S. Kubono ${ }^{2}, M$.

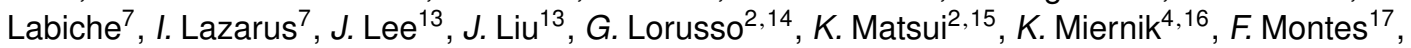
B. Moon ${ }^{18}$, A.I. Morales ${ }^{1}$, N. Nepal ${ }^{10}$, S. Nishimura ${ }^{2}$, R. D. Page ${ }^{8}$, V. H. Phong ${ }^{2,22}$, Z. Podolyak ${ }^{19}$, V. F. E. Pucknell ${ }^{7}$, B. C. Rasco ${ }^{4}$, P. Regan ${ }^{19,14}$, A. Riego ${ }^{6}$, B. Rubio ${ }^{1}$, K.P. Rykaczewski ${ }^{4}$, Y. Saito ${ }^{5}$, H. Sakurai ${ }^{2}, 15, Y$. Shimizu' ${ }^{2}$, J. Simpson ${ }^{7}$, P.-A. Söderström ${ }^{2}, D$. W. Stracener ${ }^{4}, T$. Sumikama ${ }^{2}, H$. Suzuki $^{2}$, J. L. Tain ${ }^{1}$, M. Takechi ${ }^{21}, H$. Takeda ${ }^{2}$, A. Tarifeño-Saldivia ${ }^{6}$, S. L. Thomas ${ }^{20}$, and $P$. Woods $^{9}$
\end{abstract}

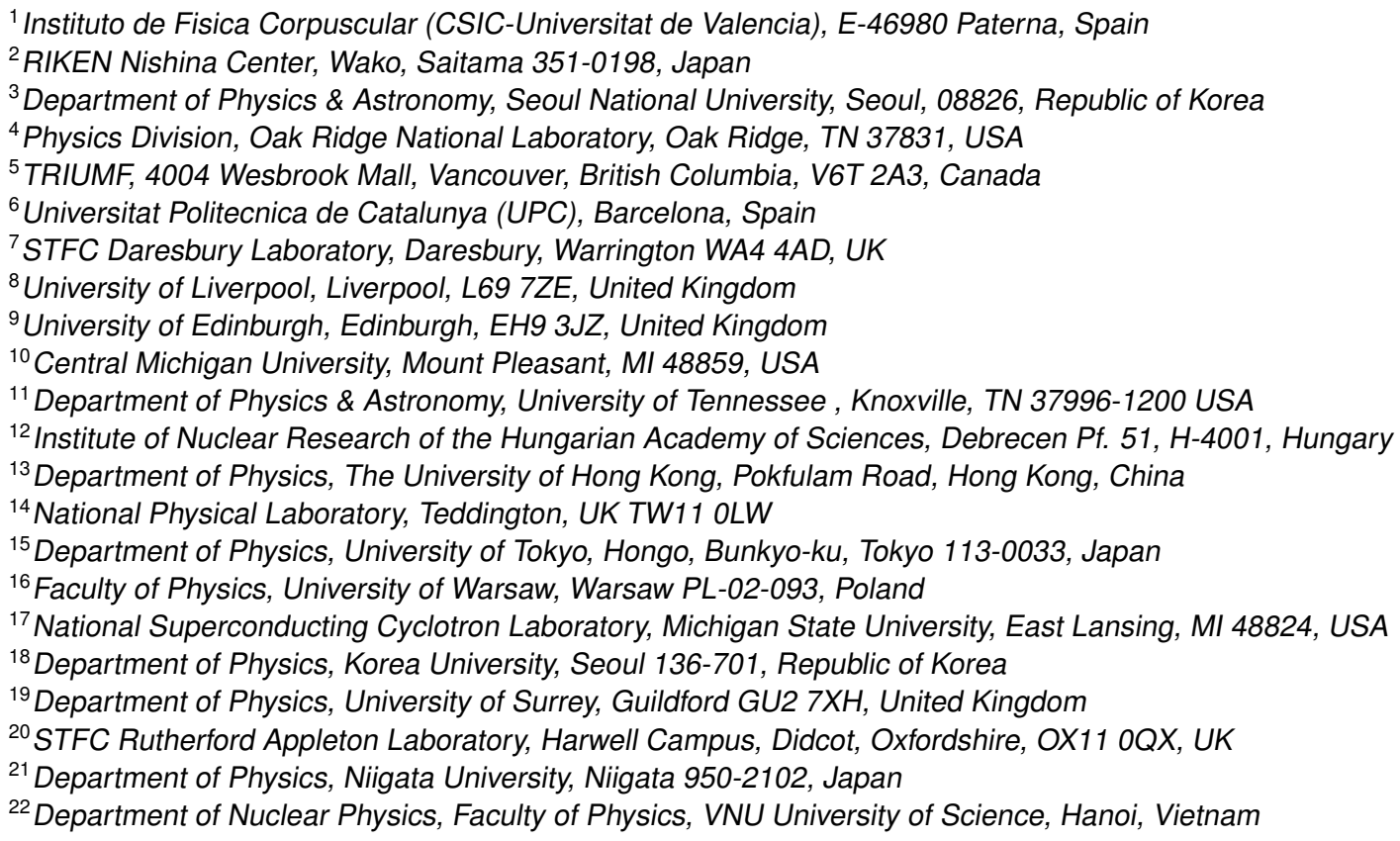

\begin{abstract}
The commissioning of a new setup for $\beta$-delayed neutron measurements was carried out successfully in November-2016, at the RIKEN Nishina Center in Japan. The $\beta$-decay half-lives and $P_{n}$ branching ratios of several isotopes in the ${ }^{78} \mathrm{Ni}$ region were measured. Details of the experimental setup and the first results are given.
\end{abstract}

${ }^{\star}$ e-mail: Alvaro.Tolosa@ific.uv.es 


\section{Motivation}

Beta-delayed neutron emission $(\beta \mathrm{n})$ is a form of radioactive decay in which an electron, an anti-neutrino and one or more neutrons are emitted. This process arises if the energy window of the decay $Q_{\beta}$ is greater than the neutron separation energy $S_{n}$ of the daughter. The probability in each decay of emitting neutrons is called the $P_{n}$ value.

This form of decay plays a key role in the synthesis of chemical elements in the Universe via the rapid neutron capture process, or r-process [1]. The r-process proceeds far from the valley of nuclear stability, and leads to very neutron-rich nuclei that then decay to the line of stability. Most of these nuclei are $\beta$ n emitters. The initial abundance distribution is shaped by the decay half-life $T_{1 / 2}$, but the neutron emission affects the final abundances in two ways: on the one hand it shifts the decay path to lower masses and on the other hand it provides a source of neutrons for late captures that will shift it in the opposite way[2]. Thus, a good knowledge of $P_{n}$ and $T_{1 / 2}$ values of the nuclei between the line of stability and the r-process path is needed for a correct understanding of the observed abundances.

Besides the astrophysical interest, the measurement of new $P_{n}$ values and half-lives is also useful for refining nuclear structure models, constraining parameters of the models and testing $\beta$-strength functions [3]. Our current understanding of the process of $\beta$-delayed multiple neutron emission, as well as the competition between different open channels (gamma, one-neutron, two-neutron, etc), is incomplete because of the scarcity of experimental data [4].

With these ideas in mind, the BRIKEN (Beta-delayed-neutron-measurements at RIKEN) [5] collaboration was established, with the aim of greatly improving the quantity and quality of data the decays of very neutron-rich nuclei close to the r-process path.

\section{Experimental setup}

A detection system for direct measurements of $\beta$ n-decay has been installed at the Radioactive Isotope Beam Factory (RIBF) in RIKEN, which currently provides the highest intensities of the most neutron-rich nuclei produced by in-flight fission.

The detection system is composed of the Advanced Implantation Detector Array (AIDA) [6], and a neutron counter. It is placed at the F11 focal plane of the BigRIPS+ZeroDegree spectrometer [7], which serves to identify the isotopes that are implanted in AIDA.

The mission of AIDA is to register ion implantations and the subsequently emitted $\beta$ particles. It consists of a stack of six Si DSSDs. There are two different electronic branches, for high- and low-energy events respectively. Offline, after consistency checks, the high energy events are identified as implants, and low energy events as $\beta$ particles. The highly segmented silicon strip detectors grant a high detection efficiency and minimize accidental implant-beta correlations. A digital eventless data acquisition system (DAQ) reads out the individual strips (1536 in total) for further processing.

\subsection{The neutron counter}

The neutron counter surrounding AIDA consists of a large polyethylene (PE) matrix with an array of $140{ }^{3} \mathrm{He}$ tubes, owned by several collaborating laboratories, including ORNL, UPC-Barcelona, GSI-Darmstadt and RIKEN. The neutron energies are moderated in collisions in the PE and the neutrons are absorbed by the ${ }^{3} \mathrm{He}$ in an exothermic reaction. The energy deposited in the tube produces 
a signal that is amplified and acquired by a digitizer working in self-triggering mode. The data are recorded by the eventless DAQ system Gasific developed at IFIC-Valencia [8].

The PE moderator geometry was optimized [9] in order to reach a high detection efficiency, that is as constant as possible for neutron energies from $1 \mathrm{keV}$ to few $\mathrm{MeV}$. The latter is required to minimize systematic errors in the derived $P_{n}$ for decays with different neutron energy distributions.

Given the very large number of ${ }^{3} \mathrm{He}$ tubes and their different characteristics (length, pressure, size) a parametrized Monte Carlo optimization algorithm was developed in order to determine their best position inside the matrix, and achieve a high and flat neutron efficiency. The configuration finally chosen includes also two Clover-type HPGe detectors. This adds high-resolution $\gamma$-ray spectroscopy capability with the selectivity provided by neutron tagging, giving additional nuclear structure information.

The GEANT4 [10] simulated efficiency was crosschecked using a ${ }^{252} \mathrm{Cf}$ neutron source. The experimental efficiency for this source is $61 \pm 2 \%$, which is in good agreement with the simulated efficiency of $62 \%$. This corresponds to an average efficiency of about $68 \%$ for neutrons below $1.5 \mathrm{MeV}$.

\section{Measurements}

The BRIKEN setup received the first radioactive beam on November 2016. A high intensity (40 pnA) primary beam of ${ }^{238} \mathrm{U}$, with energy $345 \mathrm{MeV} / \mathrm{u}$, hit a $5 \mathrm{~mm}$ Be target producing a secondary beam of exotic neutron-rich nuclei. The BigRIPS spectrometer setting was centred on ${ }^{76} \mathrm{Ni}$. Figure 1 shows an identification plot of the accumulated implants during the experiment.

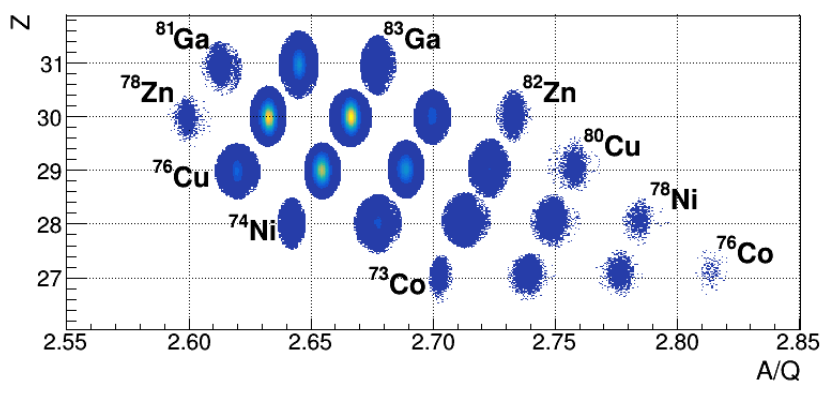

Figure 1. Particle identification plot of the implanted ions. The $\mathrm{x}$-axis of the plot represents the particle mass over its charge, and the y-axis represents the atomic number $\mathrm{Z}$. Given the high energy during the identification, the atoms are completely stripped of their electrons and their charge coincides with their atomic number.

Using these data, specific software was developed using the ROOT package [11] for merging offline the data from the three independently running but synchronized DAQs: BigRIPS, AIDA and BRIKEN. This software sorts the input data by time stamp, and at the same time includes in the output stream time-correlated data for preselected parameters; also, at this point the identification (atomic number $\mathrm{Z}$ and mass over charge ratio $\mathrm{A} / \mathrm{Q}$ ) of the implant is assigned by time coincidence with BigRIPS events. The output can be used directly in the analysis procedure where complex correlations, vetoes and tags can be applied. Figure 2 shows some of the correlated spectra that can be built using this software.

The $P_{n}$ value, the number of parent decays, and eventually the half-lives, are obtained from a simultaneous fit of the implant-decay curves using the solution of the Bateman equations [12]. The neutron efficiency is a fixed input parameter. The usual assumption that the $\beta$ efficiency is independent of the species of nucleus in the decay chain, and thus can be ignored, was tested and it was found that it requires a demanding minimization of thresholds for $\beta$ signals in AIDA. 

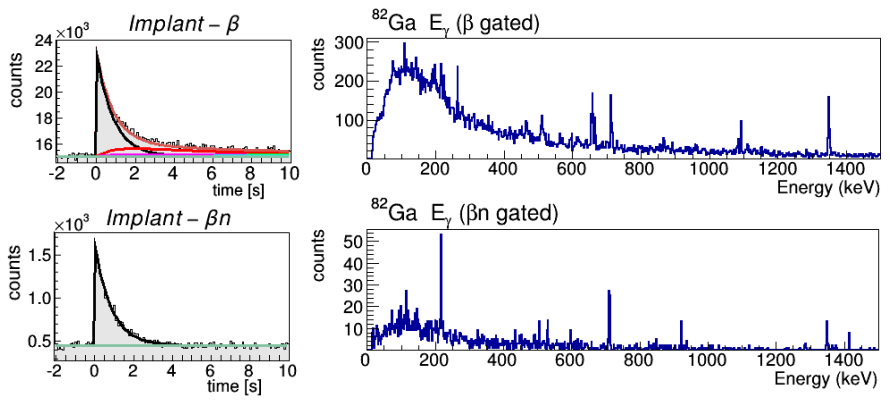

Figure 2. For each identified isotope (here ${ }^{82} \mathrm{Ga}$ ), the implantation-decay time histogram can be built from the merger output without (top left) or with $\beta$-neutron coincidence time window (bottom left). Gamma-ray energy spectra in coincidence with $\beta$ particles (top- and bottom-right) can be built integrating over a range of implantation-decay times.

\section{Results and conclusions}

A total of $15 P_{n}$ values could be determined for $\mathrm{Ni}, \mathrm{Cu}, \mathrm{Zn}$ and $\mathrm{Ga}$ isotopes. Good agreement was found with values in literature [13] for ${ }^{76} \mathrm{Cu},{ }^{77} \mathrm{Cu}$ and ${ }^{82} \mathrm{Ga}$, where there are several existing measurements, thus confirming the reliability of the data. For the first time $P_{n}$ values were determined for ${ }^{76,77} \mathrm{Ni}$ and ${ }^{80} \mathrm{Cu}$. In the decay of ${ }^{78} \mathrm{Ni}$ a total of $29 \beta$-neutron correlations were detected for the first time. More precise results were obtained for the remaining cases, some significantly different from previously published values. Overall these results demonstrate the good performance of the setup.

In May-June 2017, the first experimental BRIKEN campaign took place successfully. It covered part of the currently approved proposals. Although the data are still in the preliminary stages of the analysis we can report that several tens of new $P_{1 n}$ and $P_{2 n}$ values will be determined for the first time. Additional experiments will follow in the near future.

\section{Acknowledgement}

This work has been supported by the Spanish MINECO under grants FPA2011-24553, FPA2011-28770-C03-03, FPA2014-52823-C2-1/2, SEV-2014-0398; by STFC (UK); by FP7/EURATOM Contract No. 605203; by JSPS KAKENHI grant No. 17H06090; by NSERC grants SAPIN-2014-00028 and RGPAS 462257-2014 at TRIUMF; by U.S. DOE grant DE-AC05-00OR22725; by Polish NSC UMO-2015/18/E/ST2/002. Work partially done within IAEA-CRP for Beta Delayed Neutron Data.

\section{References}

[1] E. M. Burbidge, G. R. Burbidge, W. A. Fowler \& F. Hoyle. Rev. Mod. Phys. 29, 547 (1957)

[2] A. Arcones \& G. Martinez-Pinedo. Phys. Rev. C 83, 045809 (2011).

[3] P. Moller, B. Pfeiffer, and K.-L. Kratz, Phys. Rev. C 67, 055802 (2003)

[4] R. M. Mumpower et al. Phys. Rev C 94, 064317 (2016)

[5] https://www.wiki.ed.ac.uk/display/BRIKEN/Home

[6] T. Davinson et al., http://www2.ph.ed.ac.uk/ td/AIDA/

[7] T. Kubo et al., Prog. Theor. Exp. Phys. 2012, 03 C003 (2012)

[8] J. Agramunt et al., Nucl. Instrum. Methods Phys. Res. A 807, 69 (2016)

[9] A. Tarifeno-Saldivia et al., J. Instrum. 12, 04006 (2017)

[10] S. Agostinelli, et al. Nucl. Instrum. Methods Phys. Res., Sect. A, 506, 250 (2003)

[11] R. Brun et al., Nucl. Instrum. Methods Phys. Res. A, 389, 81 (1997)

[12] K. Skrable et al., Health Physics, 27, 155 (1974)

[13] https://www.nndc.bnl.gov/ensdf/ 